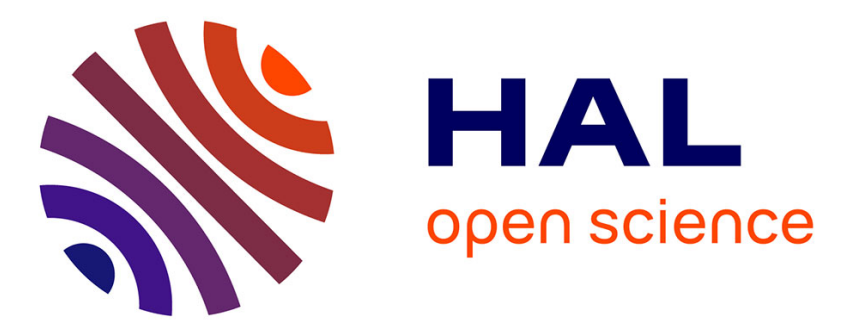

\title{
Complex mode of inheritance in holoprosencephaly revealed by whole exome sequencing
}

Charlotte Mouden, Christèle Dubourg, Wilfrid Carré, Sophie Rose, Chloé

Quélin, Linda Akloul, Géraldine Viot, Houria Salhi, Pierre Darnault, Sylvie

Odent, et al.

\section{To cite this version:}

Charlotte Mouden, Christèle Dubourg, Wilfrid Carré, Sophie Rose, Chloé Quélin, et al.. Complex mode of inheritance in holoprosencephaly revealed by whole exome sequencing. Clinical Genetics, 2016, 89 (6), pp.659-668. 10.1111/cge.12722 . hal-01259228

HAL Id: hal-01259228

https://hal-univ-rennes1.archives-ouvertes.fr/hal-01259228

Submitted on 8 Feb 2016

HAL is a multi-disciplinary open access archive for the deposit and dissemination of scientific research documents, whether they are published or not. The documents may come from teaching and research institutions in France or abroad, or from public or private research centers.
L'archive ouverte pluridisciplinaire HAL, est destinée au dépôt et à la diffusion de documents scientifiques de niveau recherche, publiés ou non, émanant des établissements d'enseignement et de recherche français ou étrangers, des laboratoires publics ou privés. 
Complex mode of inheritance in holoprosencephaly revealed by whole exome sequencing

Charlotte Mouden* ${ }^{1}$, Christèle Dubourg* ${ }^{* 1,2}$, Wilfrid Carré ${ }^{2}$, Sophie Rose ${ }^{3}$, Chloé Quelin ${ }^{4}$, Linda Akloul ${ }^{4}$, Géraldine Viot ${ }^{5}$, Houria Salhi ${ }^{6}$, Pierre Darnault ${ }^{7}$, Sylvie Odent ${ }^{1,4}$, Valérie Dupé $^{1}$ and Véronique David ${ }^{1,2}$

${ }^{1}$ UMR6290 Institut de Génétique et Développement de Rennes, Université de Rennes 1, 35043 Rennes, France

${ }^{2}$ Laboratoire de Génétique Moléculaire et Génomique, C.H.U. de Rennes, 35033 Rennes,

France

${ }^{3}$ UMR1085 Institut de Recherche sur la Santé, l'Environnement et le Travail, Université de

Rennes 1, 35043 Rennes, France

${ }^{4}$ Service de Génétique Clinique, C.H.U. de Rennes, 35200 Rennes, France

${ }^{5}$ Service de Génétique Médicale, Maternité Port Royal, 75014 Paris, France

${ }^{6}$ Foetopathologie et Anatomie Pathologique Pédiatrique, Hôpital Cochin, 75014, Paris, France

${ }^{7}$ Service de Radiologie et Imagerie Médicale, C.H.U. de Rennes, 35000 Rennes, France

* Equally contributed to the paper

Corresponding author:

Charlotte Mouden

Charlotte.mouden@,univ-rennes1.fr

+33223234397

IGDR UMR 6290 CNRS, Université de Rennes1,

Faculté de Médecine

2 Avenue du Pr Léon Bernard

35043 Rennes, France

\section{CONFLICT OF INTERESTS STATEMENT}

All authors declare that they have no conflicts of interest in the research 


\title{
ACKNOWLEDGEMENTS
}

We acknowledge and are extremely grateful to the families who participated in these research studies. We thank the Centre de Ressources Biologiques (CRB-Santé) of Rennes for managing patient samples. We also thank Michelle Ware and Marie de Tayrac for their kind help in editing the manuscript. This work was supported by Agence Nationale de la Recherche (grant no. ANR-12-BSV1-0007-01), Fondation Maladies Rares and Agence de la Biomédecine.

\begin{abstract}
Holoprosencephaly (HPE) is the most common congenital cerebral malformation, characterized by impaired forebrain cleavage and midline facial anomalies. Heterozygous mutations in 14 genes have been associated with HPE, and are often inherited from an unaffected parent underlying complex genetic bases. It is now emerging that HPE may result from a combination of multiple genetic events, rather than from a single heterozygous mutation. To explore this hypothesis, we undertook whole exome sequencing (WES) and targeted high-throughput sequencing approaches to identify mutations in HPE subjects. We report here two HPE families in which two mutations are implicated in the disease. In the first family presenting two fetuses with alobar and semi-lobar HPE, we found mutations in two genes involved in HPE, SHH and DISP1, inherited respectively from the father and the mother. The second reported case is a family with a 9-year old girl presenting lobar HPE, harbouring two compound heterozygous mutations in DISP1. Together, these cases of digenic inheritance SHH/DISP1 and autosomal recessive HPE suggest that in some families, several genetic events are necessary to cause HPE. This study highlights the complexity of HPE inheritance and has to be taken into account by clinicians to improve HPE genetic counseling.
\end{abstract}

\section{KEY WORDS}

DISP1, Holoprosencephaly, Multigenic Inheritance, SHH, Whole Exome Sequencing; 


\section{INTRODUCTION}

Holoprosencephaly (HPE) is the most frequent cerebral malformation, with an occurrence of approximately 1 in 250 embryos and 1.31 in 10,000 births (1). HPE is characterized by a failure to define the midline of the forebrain and midface, with different degrees of severity from a lobar brain to alobar forms associated with cyclopia. Mild manifestations or microforms include ocular hypotelorism, microcephaly and a single central maxillary incisor (2). The mode of inheritance of HPE has been extensively discussed in the literature, and several genetic models have been proposed: autosomal dominant transmission, autosomal recessive transmission or association of mutations in multiple genes (2-5). All these studies point out a strong genetic heterogeneity, with several causative genes identified (Table 1). It mostly implicates heterozygous mutations in SHH, ZIC2, SIX3 and TGIF1, which are considered as the four major genes involved in HPE. Heterozygous mutations in the minor genes GLI2, PTCH1, DISP1, FOXH1, NODAL, TDGF1, CDON, GAS1, DLL1 and FGF8 have been identified with a lower frequency $(2,6,7)$. Recently, two recessive inheritance cases of HPE have been described, implicating mutations in the gene STIL (Table 1) $(8,9)$. Importantly, these genes are all involved in signalling pathways implicated in brain development $(4,9-16)$ and alteration of SHH signalling appears to be the most common cause of HPE (17).

Although the major genes have been formally involved, their penetrance is usually incomplete with an intra-familial phenotypic variability. Actually, mutations located in these genes are inherited from a parent, asymptomatic or displaying a microform of HPE, in 70\% of the cases (2). For example, the same $S H H$ mutation can be found in individuals harbouring either alobar HPE or minor forms (18). Consequently, the clinical variability could be due to abnormalities in other genes that have a function in the same or interacting signalling pathways $(19,20)$.

This is strongly supported by the description of mouse models carrying mutations in two genes of the same or different signalling pathways. For example, whereas Gas1-/- mutant mice exhibit partial fusion of the medial nasal processes and Shh+/- mice appear normal, Gas1-/-;Shh+/- mice embryos display complete fusion of the medial nasal processes (21), reminiscent of a HPE phenotype. Such examples of animal models are numerous and all 
support the hypothesis that HPE could be due to a cumulative partial inhibition of signalling pathways implicated in forebrain development $(6,22)$.

However, there are only a few examples in the literature that suggest that HPE could be related to combined failures of several HPE genes in human (Table 2), including patients with co-occurring mutations or deletions in SHH/TGIF1 and SHH/ZIC2 (23). Nevertheless, sequencing of the four major genes (SHH, ZIC2, TGIF1, SIX3) in large HPE cohorts has not allowed to validate this hypothesis $(2,5,14)$. Thus, the mode of inheritance of HPE is still unclear.

Identifying more genes in families in which polygenic inheritance is suspected would be very beneficial to understand the pathogenic mechanism of this developmental disorder. This is now facilitated by the recent development of next generation sequencing technologies (20). In this study, we performed whole exome sequencing (WES) in a family where the father carries a mutation in SHH, transmitted to 3 fetuses with semi-lobar and alobar HPE. We hypothesized that the fetuses have all inherited a second mutation in another gene from the mother. This original strategy was powerful as it revealed a second mutation in DISP1 shared by the mother and the HPE fetuses. This family is the first one in which mutations in the two HPE-associated genes, SHH and DISP1, have been identified. Furthermore using a targeted NGS method, the involvement of DISP1 in HPE was reinforced by the observation of two DISP1 compound heterozygous mutations in another HPE family. These results support the complexity of HPE inheritance and raise important questions about how clinicians should consider the inheritance mode of HPE.

\section{MATERIALS AND METHODS}

\section{Patients and samples}

Patients presenting midline abnormalities and suspicion of holoprosencephaly were referred from the Centre Hospitalier Universitaire of Rennes (France) and the Hôpital Cochin (Paris, France) through the network of reference centres for developmental anomalies and malformation syndromes (CLAD centres). Patients and parents blood and tissue samples were obtained from the processing of biological samples through the Centre de Ressources Biologiques (CRB) Santé of Rennes BB-0033-00056 (http://www.crbsante-rennes.com). The research protocol was conducted under French legal guidelines and fulfilled the requirements 
of the local institutional ethics committee. The parents of the photographed patient assented to include photographs in a scientific publication.

\section{F1 family}

The mother II2 had three terminations of pregnancy with fetuses (III1, III2 and III3) harbouring semi-lobar or alobar HPE (Fig. 1a and Table 3a). The F1 family also includes two other healthy girls III4 and III5, being 12 and 11 years old respectively. The father II1, the grandfather I1 and the uncle II3 all present microcephaly. This latter had 4 children among which 3 (III6, III7 and III9) harbour microcephaly and minor facial midline malformations (hypotelorism). The father II1 also had two healthy siblings, II5 and II6 (Table 3a). DNA was available for all family members except II4.

\section{F2 family}

The F2 family includes three members, the two healthy parents I1 and I2 and their 9-year old girl II1 who displayed a lobar HPE (Fig. 2 and Table 3b). She has two healthy siblings but their DNA was not available. Pregnancy was normal. A wide cleft palate was observed at birth, which was surgically treated. II1 was then referred to genetic counselling at 5-and-halfyears old for psychomotor retardation (started walking at 21 months and had a language delay) and learning difficulties. On clinical examination, she weighed $16.5 \mathrm{~kg}(-1$ standard deviation), was $109.5 \mathrm{~cm}$ tall, and had microcephaly (-2SD). She presented facial dysmorphy with flat face, short nose, small mouth and hypotelorism (Figs. 2c and 2d). At the sight of these clinical observations, we recommended a molecular diagnosis of HPE. Molecular testing of $18 \mathrm{HPE}$ genes was performed on DNA of the daughter II1, revealing the presence of two compound heterozygous mutations in the minor HPE gene DISP1. Subsequently, to confirm the presence of HPE, MRI was performed and showed a mild form of lobar HPE with a very localized fusion of hemispheres in the forebrain (Fig. 2b).

\section{Whole Exome Sequencing}

WES was performed by the Genoscope on the DNA of the two parents II1 and II2 and one of the fetuses III2 in F1 family, using "SeqCap EZ Exome v3.0" capture (Roche) on HiSeq $^{\mathrm{TM}} 2000$ platform (Illumina). Exomes were homogenously sequenced with a mean coverage of $93 \%$ of the targeted bases with read depth greater than $20 \mathrm{X}$, and an overall mean 
depth of coverage of 112X. Bioinformatic analyses were conducted with the Illumina pipeline analysis CASAVA 1.8. The sequenced reads were aligned on the reference human genome 19 (hg19) with Eland v2.0 before variant calling (SNV and INDEL) with the CASAVA suite. Resulting variants were annotated using ANNOVAR v2.0 (http://www.openbioinformatics.org/annovar/annovar_filter.html). Variants population frequencies were extracted from three different public databases (the Exome Sequencing Project (ESP6500), the 1000 Genomes Project $(1000 \mathrm{~g}, 2014)$ and the Exome Aggregation Consortium (ExAC02)). Several bioinformatics predictions tools were used to predict conservation (GERP++, PhyloP, SiPhy, and PhastCons Elements 46-way) (24-27) and deleterious effect of SNVs and INDELs (SIFT, PolyPhen-2 HDIV and HVAR, LRT, Mutation Taster, Mutation Assessor, FATHMM, Radial SVM, LR and CADD) (28-34). Visual inspection of candidate variants was performed with Integrative Genome Viewer (IGV, Broad Institute).

\section{Targeted high-throughput sequencing}

Targeted NGS was performed using Ion Torrent technology (Life Technologies) on DNA from the girl II1 in F2 family. Two pools of 711 primer pairs were designed (Ion Ampliseq technology, Life Technologies) to sequence all the exons of a panel of 18 genes involved in HPE or candidates (SHH, ZIC2, TGIF1, SIX3, DISP1, CDON, GAS1, SUFU, FGF8, FGFR1, NODAL, HHAT, SUFU, TDGF1, PTCH1, FOXH1, SOX2, DLL1) and 2 SHH expression regulatory regions, spanning $111 \mathrm{~kb}$. Libraries were sequenced with Ion $\mathrm{PGM}^{\mathrm{TM}}$ System (Life Technologies). A PGM-specific pipeline incorporated in the Ion Torrent server (Torrent Suite version 4.0.2; Life Technologies) was used to perform the following steps: reads alignment on hg19, targeted regions coverage analysis, filtering and removal of poor signal reads. Variant calling was performed with the Ion Torrent Variant Caller version 4.0. Mutations were annotated using ANNOVAR v2.0 as described for WES analysis and with Alamut software (Interactive Biosoftware).

\section{Sanger sequencing}

Sanger sequencing in F1 and F2 families assessed the intrafamilial segregation of the candidate mutations found by NGS. This was done using the BigDye terminator cycle sequencing kit (Applied Biosystems) on an ABI3130xl sequencer (Applied Biosystems) and analysed using SeqScape software v2.6 (Life Technologies). 


\section{Functional validation of the $S H H$ p.Pro347GIn mutation}

The human SHH cDNA (RefSeq NM_000193) was cloned in a pMSCVneo (Clontech) vector. A mutated plasmid containing the $\mathrm{SHH}$ p.Pro347Gln mutation was obtained by site-directed mutagenesis using the QuikChange XL Site-directed Mutagenesis Kit (Stratagene, La Jolla, USA). Plasmids containing cDNA of SHH WT or SHH p.Pro347Gln were transfected in C3H10T1/2 cells using Transfast (Promega). Six days later, the alkaline phosphatase activity was measured as previously described (35), reliable to the differentiation of C3H10T1/2 into osteoblasts under SHH action.

\section{RESULTS}

\section{F1 family}

In the F1 family, we showed by Sanger sequencing that a p.Pro347Gln mutation in $S H H$ (c.1040C $>$ A of RefSeq NM_000193) was present in fetuses III1, III2 and III3 inherited from the father II1 (Fig. 1a). It was inherited from the grandfather I1, and was also transmitted to the uncle II3, displaying microcephaly and hypotelorism. This uncle transmitted the SHH mutation to 3 children (III6, III7 and III9), also harbouring microcephaly and hypotelorism. WES analysis validated the known heterozygous $S H H$ mutation in the father II1 presenting microcephaly and hypotelorism, and in the HPE fetus III2.

The deleterious effect of the $S H H$ p.Pro347Gln mutation was evaluated using an adaptation of a cell-based assay previously described (35). The efficiency of SHH signalisation in presence of the mutation was evaluated by quantifying the SHH-dependent differentiation of mesenchymal cells (C3H10T1/2) into osteoblasts. This was assessed by measuring the activity of the alkaline phosphatase (ALP) in C3H10T1/2 cells, 6 days after transfection with the pMSCVneo plasmids containing either cDNA of SHH WT or cDNA of SHH p.Pro347Gln. The ALP activity of the cells expressing the mutated $S H H$ was $0,04 \mu \mathrm{U}$ by $\mu \mathrm{g}$ of protein extract, while it was $1.31 \mu \mathrm{U} / \mu \mathrm{g}$ in the protein extract from cells expressing $S H H$ WT, meaning that $\mathrm{C} 3 \mathrm{H} 10 \mathrm{~T} 1 / 2$ cells failed to undergo osteoblastic differentiation under action of mutated SHH. This strongly reflects the deleteriousness of the p.Pro347Gln mutation (Fig. 1b). 
Based on the assumption that HPE observed in the fetus III2 resulted from the association of the SHH mutation with another mutation, variants were filtered according to this inheritance pattern. Only mutations inherited from the mother II2 were selected in the child III2. Intronic variants were filtered out, as well as synonymous variants and those that had a population allele frequency over 1\% in any of the three public databases $1000 \mathrm{~g}$, ESP6500, and ExAC02. Thirty-four variants were selected, and a prioritization was performed using the cumulative predictions of ten bioinformatics tools. The 10 first-ranked candidate mutations are presented in supplementary data (Fig. S1). Most of these genes were already associated with genetic syndromes without any obvious link with forebrain development. We thus focused on a mutation in DISP1, known to be involved in HPE. This mutation is a substitution of a thymine in cytosine at location c.3287 of DISP1 (NM_032890), leading to the change of a methionine in threonine at location p.1096. This mutation was listed as rs144673025 in dbSNP database, and has a minor allele frequency of $0,55 \%$ in ESP6500, 0,1398\% in $1000 \mathrm{~g}$, and $0,6189 \%$ in ExAC02. Three bioinformatics prediction tools classified this mutation as deleterious (LRT, Mutation Taster, and FATHMM) while other tools predicted it as tolerated (PolyPhen-2, SIFT, radial SVM, LR, and Mutation Assessor) (Table 4).

We used Sanger sequencing to search for the DISP1 c.3287T $>$ C mutation in individuals III3, III4 and III5 (Fig. 1). DNA was no longer available to look for this DISP1 mutation in fetus III1. A perfect co-segregation of the two mutations, SHH p.Pro347Gln and DISP1 p.Met1096Thr was observed in HPE fetuses III2 and III3. Among the two healthy sisters, III4 has no mutation whereas III5 carries the DISP1 mutation only.

\section{F2 family}

The F2 family was screened for 18 HPE candidate genes using targeted NGS for molecular diagnosis on the DNA of the daughter II1. Two heterozygous mutations were identified in the exon 10 of DISP1: the c.1087A $>\mathrm{G}$ transition leading to a missense mutation $\mathrm{p}$.Asn363Asp and the c.1657G $>$ A transition leading to a missense mutation p.Glu553Lys. Sanger sequencing on DNA of the parents showed that the p.Asn363Asp mutation was inherited from the father I1, and the p.Glu553Lys mutation was inherited from the mother I2 (Fig. 2a).

The DISP1 p.Asn363Asp mutation was absent from public databases, and was predicted deleterious by 7 out of 10 of the prediction tools employed. The second mutation, p.Glu553Lys, was also predicted deleterious by the majority of the prediction tools, and was also absent from public sequencing databases (Table 4). According to the Alamut software 
and the other conservation scores used, these two mutations were in highly conserved regions at both the nucleotide and the amino acid level.

\section{DISCUSSION}

The genetic heterogeneity of HPE is supported by reduced penetrance and the absence of obvious genotype-phenotype correlation. This is especially true for patients carrying heterozygous $\mathrm{SHH}$ mutations, as $45 \%$ harbor microforms, $45 \%$ present severe HPE and 10\% are apparently asymptomatic (18). Using WES, we report here the first co-segregation of mutations in SHH and DISP1 with severe HPE. Our results suggest that these mutations are combining to give a severe phenotype and provide strong evidence that digenic inheritance is a significant genetic model for HPE.

\section{Relationships between SHH and DISP1 in HPE}

In this study, we have investigated one family carrying a deleterious mutation in $\mathrm{SHH}$ and displaying variable expressivity of the disease. This mutation causes the change of proline in glutamine, which results in a severe reduction of SHH activity. Although microcephaly is not a typical sign of HPE, we can consider that the $S H H$ mutation is responsible for this mild form of HPE in the present family (2). SHH mutation shows full penetrance with microcephaly whereas a second mutation in DISP1 seems to be necessary to obtain a more severe HPE in 3 fetuses. This is also supported by the finding that the mother and a clinically normal sister carried only the mutation in DISP1.

In this study we also describe the first HPE case with compound heterozygous mutations in DISP1. Altogether, we find 3 different mutations (Fig. 3) in conserved regions of DISP1, including one in the Sterol Sensing Domain (SSD). The exact role of the SSD remains unclear, although most SSD containing proteins have been implicated in intracellular trafficking $(36,37)$. These 3 missense mutations in DISP1 provide new arguments for the implication of this gene in HPE. Few other mutations in DISP1 have been previously described in patient only harbouring microforms of $\operatorname{HPE}(38,39)$. Noteworthy, all these DISP1 mutations were inherited from clinically unaffected parents. This gives evidence that additional factors are necessary to potentiate these mutations of DISP1 and to lead to HPE. As DISP1 mediates the secretion of SHH from producing cells, and allows consequent paracrine signalling (40), we hypothesized that these mutations have an impact on SHH signalling. 
Mice data also strongly reveal the implication of Disp1 in HPE (40-42). Heterozygous knockout for Disp1 are undistinguishable from the wildtype whereas Disp1 $1^{-/}$embryos do not survive beyond E9.5 because of heart development defects. They also display cyclopia reminiscent to severe HPE (43). Further analysis of these mutants permitted to show that Shh signalling was disrupted in Disp1 ${ }^{-/}$embryos, indicating that Disp1 is essential for proper Shh signalling. Thus, Disp1 is most probably critical for ventral forebrain induction through its interaction with Shh pathway. Consequently, the co-segregation of a mutation in $\mathrm{SHH}$ and a mutation in DISP1 with severe HPE strongly suggests that cumulative effects lead to severe impairment of forebrain development.

\section{Inheritance in HPE}

Some authors have proposed autosomal recessive inheritance in HPE (3, 44). However, despite systematic sequencing, homozygous mutations in the major HPE genes SHH, ZIC2 and SIX3, were never described in $\operatorname{HPE}$ cohorts $(2,5,45)$. Nonetheless some autosomal recessive cases implicating minor HPE genes (Table 1) have been reported. In 2007, a first case of recessive inheritance of TGIF1 mutations was described, with the finding of two compound heterozygous mutations (46). A loss of function homozygous mutation in FGF8 has also been identified in one consanguineous HPE family (47). More recently, hypomorphic alleles of STIL were implicated in two cases of autosomal recessive inheritance in HPE patients $(8,9)$. It was proposed that STIL had a function during early brain development linked to SHH signalling $(9,48)$. Here, we describe a first HPE patient displaying two mutated DISP1 alleles whereas the two clinically normal parents carry only one mutated allele. This strongly supports that the presence of two mutations in a minor HPE gene exacerbates the risk of developing a HPE phenotype. Furthermore, the mild phenotype of the HPE patient described in this manuscript suggests that DISP1 activity is decreased such that it leads to a significant impairing of SHH pathway responsible for mild HPE, but above the threshold that would result in severe HPE and early lethality, as indicated by the mice model (43).

Currently, single heterozygous mutations are mainly reported in HPE cases (Table 1) and believed to account for the HPE phenotype (5). But still, it cannot be excluded that a mutation in another gene may underlie the observed forebrain defects. However, double heterozygous mutations for two HPE genes were rarely reported (Table 2). This is not really surprising because HPE genes are key developmental genes, and strong deleterious mutations in two of 
these genes are probably not viable $(44,49)$. Thus, it is important to design the pipeline of WES analysis in order to avoid discarding mutations predicted to have a mildly deleterious effect in development genes. This strategy has enabled us to identify a new DISP1 mutation in a family presenting two fetuses with alobar and semi-lobar HPE associated to a deleterious mutation in SHH. The identification of these two altered genes that have functional relationships in multiple affected individuals in one family strongly supports a digenic inheritance (19).

Polygenic inheritance has now been established for more and more other complex inheritance diseases among which digenic inheritance is the simplest form $(19,20)$. This mode of inheritance was reported in Kallmann syndrome (KS) (50), characterized by a defective hormonal reproductive axis and sense of smell. This developmental pathology was firstly described as autosomal dominant or X chromosome-linked. Further studies permitted to refine the genetics of KS syndrome by describing several patients harbouring two mutations in different genes $(51,52)$, combining major and minor KS genes, and giving evidence of a digenic inheritance of KS syndrome. This was also reported in patients with Alport syndrome, presenting mutations in two different collagen IV genes (COL4A3 and COL4A4) (53). In some nephropathies, mutations in two genes encoding glomerular proteins nephrin and podocin (NPHS1 and NPHS2) were identified in several patients (54). Such a multigenic inheritance is also well described in ciliopathies like Bardet-Biedl syndrome (BBS) with many patients harbouring mutations in two or more BBS genes (55). These cases illustrate how, in complex syndromes with variable severity, polygenic inheritance plays a role in the clinical expression of the disease. This is particularly relevant when there is functional relationships between mutated genes, as this is the case for HPE genes (44). A first online database dedicated to digenic diseases (DIDA, http://dida.ibsquare.be/) is now available (20). The cases described in our manuscript fully meet the required criteria to be included in DIDA database (20).

By describing new HPE families with no classical autosomal dominant inheritance, our work refines the genetic bases of HPE. This discovery has significant implications for genetic counseling especially for risk assessment of patient relatives. Clinical geneticists have to be aware of such different patterns of heritability, and WES or at least sequencing of a large panel of HPE genes, should be performed to establish a molecular diagnosis.

\section{REFERENCES}


1. Leoncini E, Baranello G, Orioli IM et al. Frequency of holoprosencephaly in the International Clearinghouse Birth Defects Surveillance Systems: searching for population variations. Birth Defects Res A Clin Mol Teratol 2008: 82: 585-591.

2. Mercier S, Dubourg C, Garcelon N et al. New findings for phenotype-genotype correlations in a large European series of holoprosencephaly cases. J Med Genet 2011: 48: 752-760.

3. Barr M, Jr., Cohen MM, Jr. Autosomal recessive alobar holoprosencephaly with essentially normal faces. Am J Med Genet 2002: 112: 28-30.

4. Ming JE, Kaupas ME, Roessler E et al. Mutations in PATCHED-1, the receptor for SONIC HEDGEHOG, are associated with holoprosencephaly. Hum Genet 2002: 110: 297-301.

5. Roessler E, Velez JI, Zhou N, Muenke M. Utilizing prospective sequence analysis of SHH, ZIC2, SIX3 and TGIF in holoprosencephaly probands to describe the parameters limiting the observed frequency of mutant genexgene interactions. Mol Genet Metab 2012: 105: 658-664.

6. Mercier S, David V, Ratié L et al. NODAL and SHH dose-dependent double inhibition promotes an HPE-like phenotype in chick embryos. Dis Model Mech 2013: 6: 537-543.

7. Solomon BD, Gropman A, Muenke M. Holoprosencephaly Overview. In: Pagon RA, Adam MP, Ardinger HH et al., eds. GeneReviews(R). Seattle (WA), 1993.

8. Kakar N, Ahmad J, Morris-Rosendahl DJ et al. STIL mutation causes autosomal recessive microcephalic lobar holoprosencephaly. Hum Genet 2015: 134: 45-51.

9. Mouden C, de Tayrac M, Dubourg C et al. Homozygous STIL Mutation Causes Holoprosencephaly and Microcephaly in Two Siblings. Plos One 2015: 10: e0117418.

10. De La Cruz JM, Bamford RN, Burdine RD et al. A loss-of-function mutation in the CFC domain of TDGF1 is associated with human forebrain defects. Hum Genet 2002: 110: 422428.

11. Arauz RF, Solomon BD, Pineda-Alvarez DE et al. A hypomorphic allele in the FGF8 gene contributes to holoprosencephaly and is allelic to gonadotropin-releasing hormone deficiency in humans. Mol Syndromol 2010: 1: 59-66.

12. Ribeiro LA, Quiezi RG, Nascimento A, Bertolacini CP, Richieri-Costa A. Holoprosencephaly and holoprosencephaly-like phenotype and GAS1 DNA sequence changes: Report of four Brazilian patients. Am J Med Genet 2010: 152: 1688-1694.

13. Bae GU, Domené S, Roessler E et al. Mutations in CDON, encoding a hedgehog receptor, result in holoprosencephaly and defective interactions with other hedgehog receptors. Am J Hum Genet 2011: 89: 231-240.

14. Dubourg C, David V, Gropman A et al. Clinical utility gene card for: Holoprosencephaly. Eur J Hum Genet 2011: 19: preceeding 118-120.

15. Dupé V, Rochard L, Mercier S et al. NOTCH, a new signaling pathway implicated in holoprosencephaly. Hum Mol Genet 2011: 20: 1122-1131.

16. Ratié L, Ware M, Barloy-Hubler F et al. Novel genes upregulated when NOTCH signalling is disrupted during hypothalamic development. Neural Dev 2013: 8: 25.

17. Cohen MM, Jr. Hedgehog signaling update. Am J Med Genet A 2010: 152A: 1875-1914.

18. Solomon BD, Bear Ka, Wyllie a et al. Genotypic and phenotypic analysis of 396 individuals with mutations in Sonic Hedgehog. J Med Genet 2012: 49: 473-479.

19. Schaffer AA. Digenic inheritance in medical genetics. J Med Genet 2013: 50: 641-652.

20. Gazzo AM, Daneels D, Cilia E et al. DIDA: A curated and annotated digenic diseases database. Nucleic Acids Res 2015.

21. Allen BL, Tenzen T, McMahon AP. The Hedgehog-binding proteins Gas1 and Cdo cooperate to positively regulate Shh signaling during mouse development. Genes Dev 2007: 21: $1244-1257$.

22. Krauss RS. Holoprosencephaly: new models, new insights. Expert Rev Mol Med 2007: 9: $1-17$. 
23. Nanni L, Ming JE, Bocian $\mathrm{M}$ et al. The mutational spectrum of the Sonic Hedgehog gene in holoprosencephaly: SHH mutations cause a significant proportion of autosomal dominant holoprosencephaly. Hum Mol Genet 1999: 8: 2479-2488.

24. Siepel A, Bejerano G, Pedersen JS et al. Evolutionarily conserved elements in vertebrate, insect, worm, and yeast genomes. Genome Res 2005: 15: 1034-1050.

25. Garber M, Guttman M, Clamp $M$ et al. Identifying novel constrained elements by exploiting biased substitution patterns. Bioinformatics 2009: 25: 54-62.

26. Davydov EV, Goode DL, Sirota M et al. Identifying a high fraction of the human genome to be under selective constraint using GERP++. PLoS Comput Biol 2010: 6.

27. Pollard KS, Hubisz MJ, Rosenbloom KR, Siepel A. Detection of nonneutral substitution rates on mammalian phylogenies. Genome Res 2010: 20: 110-121.

28. Ng PC, Henikoff S. Predicting deleterious amino acid substitutions. Genome Res 2001: 11: 863-874.

29. Chun S, Fay JC. Identification of deleterious mutations within three human genomes Identification of deleterious mutations within three human genomes. Genome Res 2009: $1553-1561$.

30. Reva B, Antipin Y, Sander C. Predicting the functional impact of protein mutations: Application to cancer genomics. Nucleic Acids Res 2011: 39: 37-43.

31. Adzhubei I, Jordan DM, Sunyaev SR. Predicting Dunctional Effect of Human Missense Mutations Using PolyPhen-2. Curr Protoc Hum Genet 2013: 7.

32. Shihab Ha, Gough J, Cooper DN et al. Predicting the functional, molecular, and phenotypic consequences of amino acid substitutions using hidden Markov models. Hum Mutat 2013: 34: 57-65.

33. Dong $\mathrm{C}$, Wei $\mathrm{P}$, Jian $\mathrm{X}$ et al. Comparison and integration of deleteriousness prediction methods for nonsynonymous SNVs in whole exome sequencing studies. Hum Mol Genet 2014: 24: 2125-2137.

34. Kircher M, Witten DM, Jain P et al. A general framework for estimating the relative pathogenicity of human genetic variants. Nat Genet 2014: 46: 310-315.

35. Traiffort E, Dubourg C, Faure H et al. Functional characterization of Sonic Hedgehog mutations associated with holoprosencephaly. J Biol Chem 2004: 279: 42889-42897.

36. Kuwabara PE, Labouesse M. The sterol-sensing domain: Multiple families, a unique role? Trends Genet 2002: 18: 193-201.

37. Nakano Y, Kim HR, Kawakami a et al. Inactivation of dispatched 1 by the chameleon mutation disrupts Hedgehog signalling in the zebrafish embryo. Dev Biol 2004: 269: 381-392. 38. Roessler E, Ma Y, Ouspenskaia MV et al. Truncating loss-of-function mutations of DISP1 contribute to holoprosencephaly-like microform features in humans. Hum Genet 2009: 125: 393-400.

39. Kantarci S, Ackerman KG, Russel $\mathrm{MN}$ et al. Characterization of the chromosome 1q41q42.12 region, and the candidate gene DISP1, in patients with CDH. Am J Med Genet 2010: 10: 2493-2504.

40. Tian H, Jeong J, Harfe BD, Tabin CJ, McMahon AP. Mouse Disp1 is required in sonic hedgehog-expressing cells for paracrine activity of the cholesterol-modified ligand. Development (Cambridge, England) 2005: 132: 133-142.

41. Kawakami T, Kawcak T, Li YJ et al. Mouse dispatched mutants fail to distribute hedgehog proteins and are defective in hedgehog signaling. Development 2002: 129: 57535765 .

42. Caspary T, Garcia-Garcia MJ, Huangfu D et al. Mouse Dispatched homolog1 is required for long-range, but not juxtacrine, Hh signaling. Curr Biol 2002: 12: 1628-1632. 
43. Kawakami T, Kawcak TN, Li Y-J et al. Mouse dispatched mutants fail to distribute hedgehog proteins and are defective in hedgehog signaling. Development (Cambridge, England) 2002: 129: 5753-5765.

44. Cohen MM, Jr. Holoprosencephaly: clinical, anatomic, and molecular dimensions. Birth Defects Res A Clin Mol Teratol 2006: 76: 658-673.

45. Dubourg C, Lazaro L, Pasquier L et al. Molecular screening of SHH, ZIC2, SIX3, and TGIF genes in patients with features of holoprosencephaly spectrum: Mutation review and genotype-phenotype correlations. Hum Mutat 2004: 24: 43-51.

46. El-Jaick KB, Powers SE, Bartholin L et al. Functional analysis of mutations in TGIF associated with holoprosencephaly. Mol Cell Biol 2007: 90: 97-111.

47. McCabe MJ, Gaston-Massuet C, Tziaferi V et al. Novel FGF8 mutations associated with recessive holoprosencephaly, craniofacial defects, and hypothalamo-pituitary dysfunction. J Clin Endocrinol Metab 2011: 96: E1709-1718.

48. David A, Liu F, Tibelius A et al. Lack of centrioles and primary cilia in STIL-/- mouse embryos. Cell Cycle 2014: 13: 2859-2868.

49. Roessler E, Muenke M. The molecular genetics of holoprosencephaly. Am J Med Genet C Semin Med Genet 2010: 154C: 52-61.

50. Klein VR, Friedman JM, Brookshire GS, Brown OE, Edman CD. Kallmann syndrome associated with choanal atresia. Clin Genet 1987: 31: 224-227.

51. Canto P, Munguia P, Soderlund D, Castro JJ, Mendez JP. Genetic analysis in patients with Kallmann syndrome: coexistence of mutations in prokineticin receptor 2 and KAL1. J Androl 2009: 30: 41-45.

52. Dode C, Hardelin JP. Clinical genetics of Kallmann syndrome. Ann Endocrinol (Paris) 2010: 71: 149-157.

53. Mencarelli Ma, Heidet L, Storey $\mathrm{H}$ et al. Evidence of digenic inheritance in Alport syndrome. J Med Genet 2015: 52: 163-174.

54. Koziell A, Grech V, Hussain S et al. Genotype/phenotype correlations of NPHS1 and NPHS2 mutations in nephrotic syndrome advocate a functional inter-relationship in glomerular filtration. Hum Mol Genet 2002: 11: 379-388.

55. Katsanis N, Eichers ER, Ansley SJ et al. BBS4 is a minor contributor to Bardet-Biedl syndrome and may also participate in triallelic inheritance. Am J Hum Genet 2002: 71: 22-29. 56. Roessler E, Belloni E, Gaudenz K et al. Mutations in the human Sonic Hedgehog gene cause holoprosencephaly. Nat Genet 1996: 14: 357-360.

57. Roessler E, Belloni E, Gaudenz K et al. Mutations in the C-terminal domain of Sonic Hedgehog cause holoprosencephaly. Hum Mol Genet 1997: 6: 1847-1853.

58. Heussler HS, Suri M, Young ID, Muenke M. Extreme variability of expression of a Sonic Hedgehog mutation: attention difficulties and holoprosencephaly. Arch Dis Child 2002: 86: 293-296.

59. Brown SA, Warburton D, Brown LY et al. Holoprosencephaly due to mutations in ZIC2, a homologue of Drosophila odd-paired. Nat Genet 1998: 20: 180-183.

60. Orioli IM, Castilla EE, Ming JE et al. Identification of novel mutations in SHH and ZIC2 in a South American (ECLAMC) population with holoprosencephaly. Hum Genet 2001: 109: $1-6$.

61. Solomon BD, Lacbawan F, Mercier $\mathrm{S}$ et al. Mutations in ZIC2 in human holoprosencephaly: description of a novel ZIC2 specific phenotype and comprehensive analysis of 157 individuals. J Med Genet 2010: 47: 513-524.

62. Ramocki MB, Scaglia F, Stankiewicz P et al. Recurrent partial rhombencephalosynapsis and holoprosencephaly in siblings with a mutation of ZIC2. Am J Med Genet A 2011: 155A: 1574-1580. 
63. Wallis DE, Roessler E, Hehr U et al. Mutations in the homeodomain of the human SIX3 gene cause holoprosencephaly. Nat Genet 1999: 22: 196-198.

64. Pasquier L, Dubourg C, Blayau M et al. A new mutation in the six-domain of SIX3 gene causes holoprosencephaly. Eur J Hum Genet 2000: 8: 797-800.

65. Ribeiro LA, El-Jaick KB, Muenke M, Richieri-Costa A. SIX3 mutations with holoprosencephaly. Am J Med Genet A 2006: 140: 2577-2583.

66. Lacbawan F, Solomon BD, Roessler E et al. Clinical spectrum of SIX3-associated mutations in holoprosencephaly: correlation between genotype, phenotype and function. $\mathrm{J}$ Med Genet 2009: 46: 389-398.

67. Hehr U, Pineda-Alvarez DE, Uyanik G et al. Heterozygous mutations in SIX3 and SHH are associated with schizencephaly and further expand the clinical spectrum of holoprosencephaly. Hum Genet 2010: 127: 555-561.

68. Gripp KW, Wotton D, Edwards MC et al. Mutations in TGIF cause holoprosencephaly and link NODAL signalling to human neural axis determination. Nat Genet 2000: 25: 205208.

69. Aguilella C, Dubourg C, Attia-Sobol J et al. Molecular screening of the TGIF gene in holoprosencephaly: identification of two novel mutations. Hum Genet 2003: 112: 131-134.

70. Rahimov F, Ribeiro LA, de Miranda E, Richieri-Costa A, Murray JC. GLI2 mutations in four Brazilian patients: how wide is the phenotypic spectrum? Am J Med Genet A 2006: 140: 2571-2576.

71. Ribeiro LA, Murray JC, Richieri-Costa A. PTCH mutations in four Brazilian patients with holoprosencephaly and in one with holoprosencephaly-like features and normal MRI. Am J Med Genet A 2006: 140: 2584-2586.

72. Roessler E, Du YZ, Mullor JL et al. Loss-of-function mutations in the human GLI2 gene are associated with pituitary anomalies and holoprosencephaly-like features. Proc Natl Acad Sci U S A 2003: 100: 13424-13429.

73. Bertolacini CD, Ribeiro-Bicudo LA, Petrin A, Richieri-Costa A, Murray JC. Clinical findings in patients with GLI2 mutations--phenotypic variability. Clin Genet 2012: 81: 70-75.

74. Roessler E, Ouspenskaia MV, Karkera JD et al. Reduced NODAL signaling strength via mutation of several pathway members including FOXH1 is linked to human heart defects and holoprosencephaly. Am J Hum Genet 2008: 83: 18-29.

75. Roessler E, Pei W, Ouspenskaia MV et al. Cumulative ligand activity of NODAL mutations and modifiers are linked to human heart defects and holoprosencephaly. Mol Genet Metab 2009: 98: 225-234.

76. Bennett H, Presti A, Adams D et al. A prenatal presentation of severe microcephaly and brain anomalies in a patient with novel compound heterozygous mutations in the STIL gene found postnatally with exome analysis. Pediatr Neurol 2014: 51: 1-3.

77. Lacbawan F, Solomon BD, Roessler E et al. Clinical spectrum of SIX3-associated mutations in holoprosencephaly: correlation between genotype, phenotype and function. $\mathrm{J}$ Med Genet 2009: 46: 389-398. 


\section{LEGENDS}

(a)
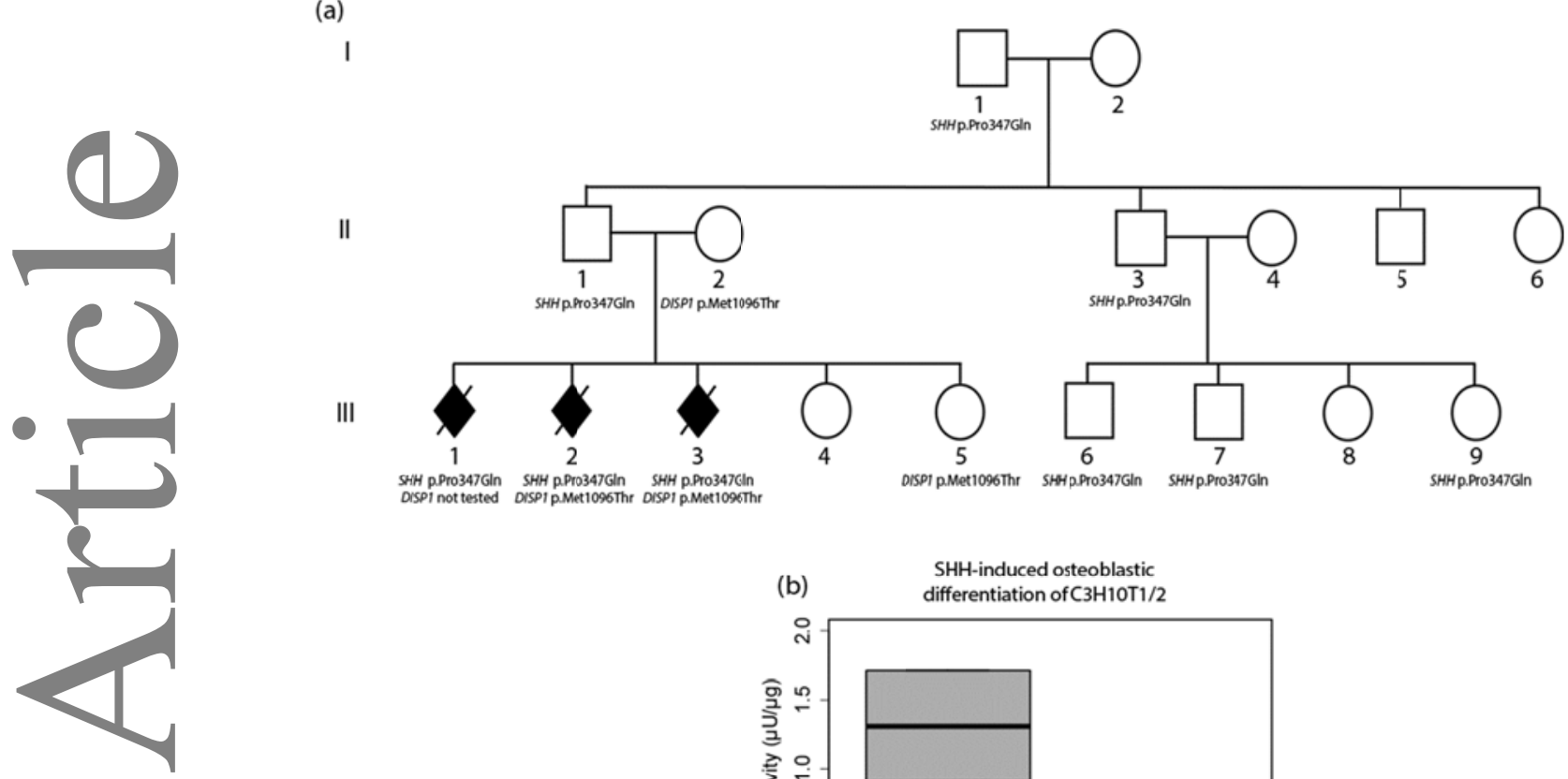

SHH p.Pro347GIn SHH P.Pro347GIn SHH P.PTO347Gln

SHHP.Pro347GI

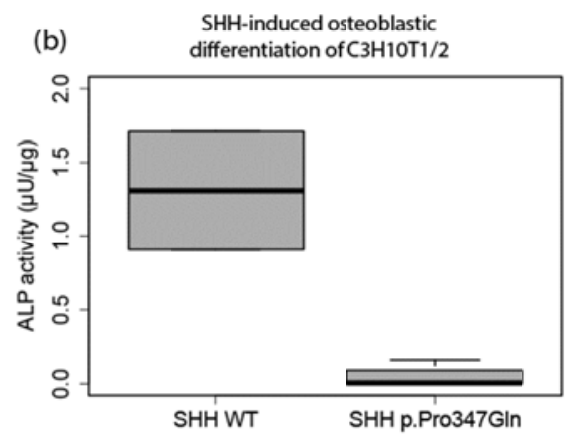

Figure 1. Pedigree of F1 family and functional test of the $S H H$ p.Pro347GIn mutation

(a) Black symbols refer to HPE subjects. Mutations were represented in proteic nomenclature. The F1 family consisted of 17 members. Nine of them harbor the SHH p.Pro347Gln mutation, inherited from the paternal grand-father I1. In addition to the SHH mutation, fetuses III2 and III3 have a DISP1 p.Met1096Thr mutation, transmitted by their mother II2. The fetus III1 was not tested for DISP1, as DNA quantity was insufficient. (b) Alkaline Phosphatase (ALP) activity measured in total protein extracts from $\mathrm{C} 3 \mathrm{H} 10 \mathrm{~T} 1 / 2$ cells transfected with plasmids containing SHH WT or SHH p.Pro347Gln. 
(a)
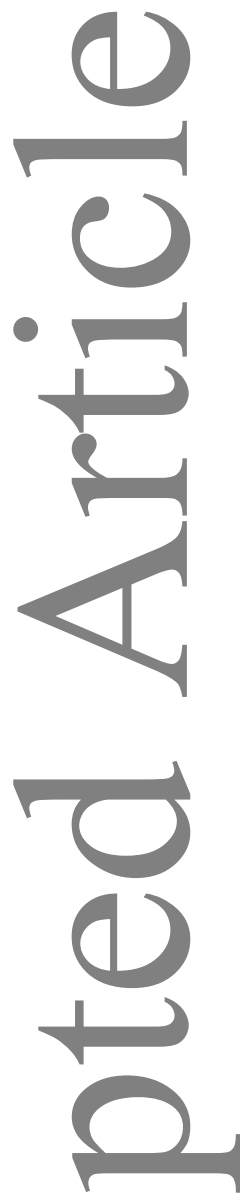

(1)
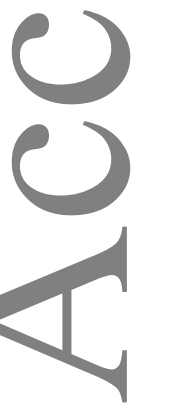
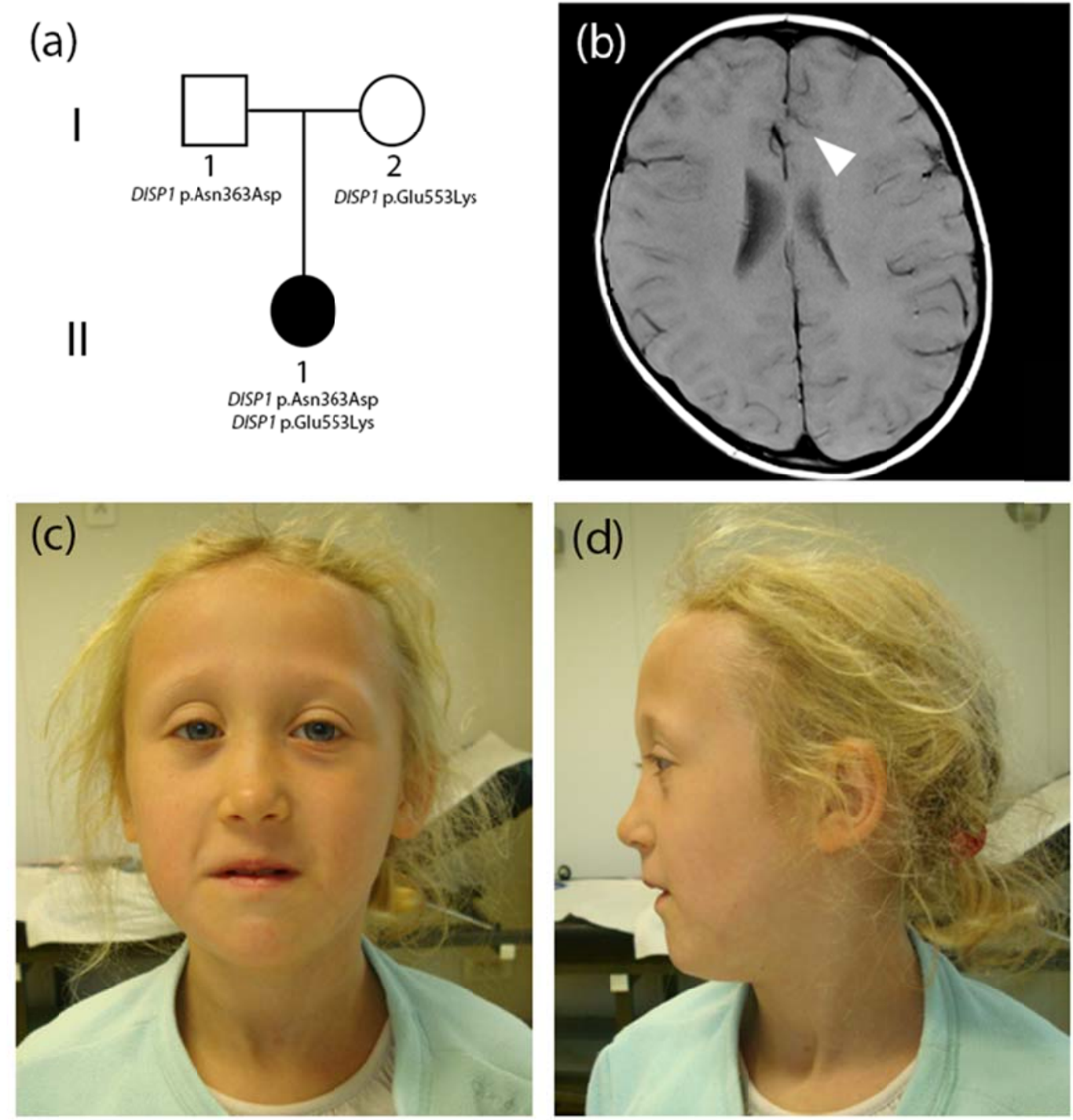

Figure 2. Pedigree of F2 family, and MRI of individual II1 of F2 family

(a) The F2 family consisted of 3 members. The daughter II1 has two DISP1 compound heterozygous mutations, DISP1 p.Asn363Asp, inherited from the father I1, and DISP1 p.Glu553Lys, inherited from the mother I2. (b) Axial brain MRI of individual II1 of F2 family, harbouring a minor form of HPE, showing a very localized fusion of the hemispheres in the forebrain (white arrow). Facial (c) and lateral (d) photographs of the daughter II1 showing mild facial malformations. 


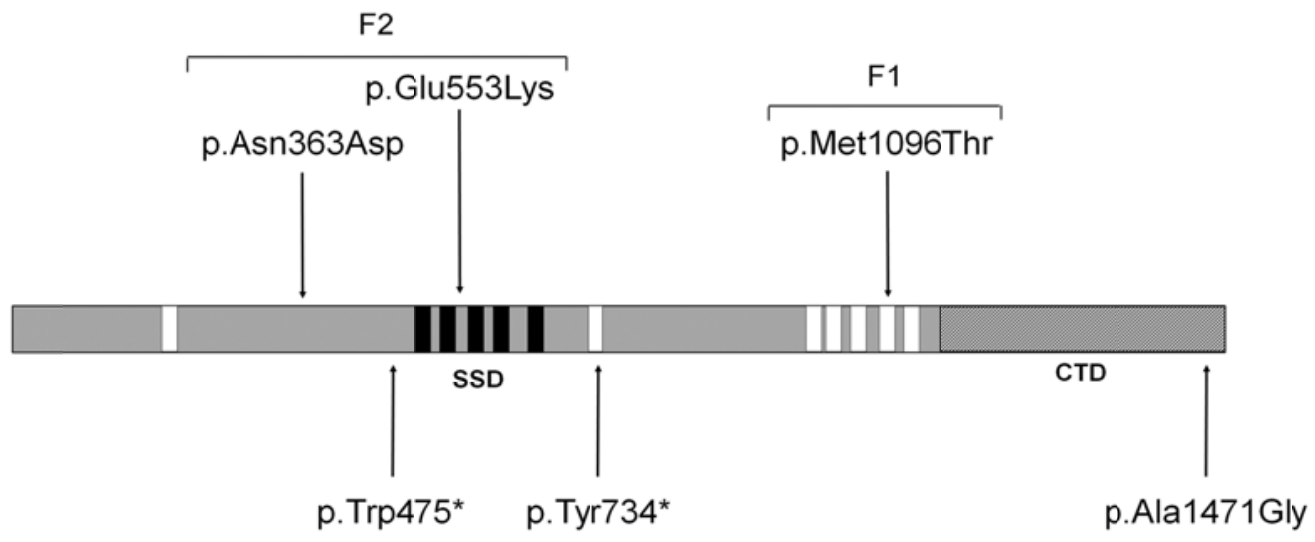

\section{Figure 3. Distribution of all DISP1 point mutations reported so far in HPE subjects}

Mutations are shown on a schematic representation of DISP1 protein. The white and black rectangles represent transmembrane helical domains, including the Sterol-Sensing Domain (SSD) from amino acid 486 to 658 (black). The hatched area is the C-terminal Domain (CTD, 360 last aminoacids of the protein). Mutations presented by families F1 and F2 are represented at the top, whereas mutations previously reported in the literature are represented at the bottom. The p.Trp475* and p.Tyr734* mutations were described by Roessler et al (38). The first mutation was present in a girl with seizures, developmental delay, midline cleft lip/palate and mild decortication, inherited from her mother. The second mutation was transmitted from a mother to her daughter who presents facial malformations: bilateral cleft lip/palate, hypotelorism, upslanting palpebral fissures and solitary maxillary central incisor. The p.Ala1471Gly mutation was reported by in a boy, born with heart abnormalities (ventricular septal defect and abnormal aorta), Bochdalek congenital diaphragmatic hernia and left-sided cleft lip with bilateral cleft palate (39). 
Table 1. Characteristics of genes and mutations implicated in holoprosencephaly.

The mutation frequencies are given in qualitative terms: "High" is for genes mutated in more than $10 \%$ of HPE cases, "Medium" for genes mutated between $10 \%$ and $1 \%$, and "Low" for genes mutated in less than 1\% of HPE cases. Mutation frequencies were calculated based on our local HPE cohort (>1000 cases). Mainly inherited: inherited mutations from a parent are predominant among the reported cases; inherited: All reported mutations are inherited from a parent; NA: inheritance information was not available. CNV or large indels encompassing whole genes are not included in this table.

\section{Table 2. Digenic inheritance in human HPE cases}

Mutations are given in proteic nomenclature, except the del18p11 which carries the TGIF1 gene off.

\section{Tables 3. Phenotypic description of families F1 and F2 members.}

(a) Phenotypic description of family F1 members. The head circumferences of individuals harboring microcephaly are given in standard deviation (SD). Facial description of fetus III3 was not available (NA). The dash means that the phenotype is normal. (b) Phenotypic description of family F2 members. M=Male, and F=Female.

\section{Table 4. Characteristics of DISP1 and SHH mutations found in F1 and F2 families}

Mutations reported in F1 family (DISP1 p.Met1096Thr; SHH p.Pro347Gln) and in F2 family (DISP1 p.Asn363Asp; DISP1 p.Glu553Lys) were annotated using ANNOVAR. Minor alleles frequencies were extracted from dbSNP build 138, in the Exome Sequencing Project containing sequencing data from 6500 exomes (ESP6500), in the 1000 Genome Project release of 2014 (1000G), and in the Exome Annotation Consortium (ExAC) containing sequencing data from 60700 exomes. Bioinformatic predictions were given by 10 predictions tools (SIFT, PolyPhen-2 HDIV and HVAR, LRT, Mutation Taster, Mutation Assessor, FATHMM, Radial SVM, LR and CADD). The cumulated predictions were given here, $\mathrm{D}=$ Deleterious, $\mathrm{P}=$ Possibly Deleterious, $\mathrm{T}=$ Tolerated. 


\section{TABLES}

\begin{tabular}{|c|c|c|c|c|c|}
\hline Gene & $\begin{array}{c}\text { Chromosomal } \\
\text { Locus }\end{array}$ & $\begin{array}{c}\text { Mutation frequency } \\
\text { in nonsyndromic } \\
\text { HPE }\end{array}$ & $\begin{array}{c}\text { Percentage of } \\
\text { Inherited mutations } \\
\text { from a parent }\end{array}$ & Zygosity state & References \\
\hline $\mathrm{SHH}$ & $7 q 36$ & High (12\%) & $70 \%$ & Heterozygous & $(5,23,45,56-58)$ \\
\hline ZIC2 & $13 q 32$ & Medium (9\%) & $30 \%$ & Heterozygous & $(5,45,59-62)$ \\
\hline SIX3 & $2 \mathrm{p} 21$ & Medium (5\%) & $70 \%$ & Heterozygous & $(5,45,63-67)$ \\
\hline TGIF1 & $18 p 11.3$ & Medium $(1,7 \%)$ & Mainly inherited & Heterozygous & $(5,68,69)$ \\
\hline PTCH1 & $9 q 22.3$ & Low & Mainly inherited & Heterozygous & $(4,70,71)$ \\
\hline TDGF1 & 3p23-p21 & Low & NA & Heterozygous & $(10)$ \\
\hline GLI2 & $2 q 14$ & Low & Mainly inherited & Heterozygous & $(70,72,73)$ \\
\hline DISP1 & $1 q 42$ & Low & Mainly inherited & Heterozygous & $(38,39)$ \\
\hline FGF8 & $10 q 24$ & Low & Mainly inherited & $\begin{array}{l}\text { Heterozygous } \\
\text { Homozygous }\end{array}$ & $(11,47)$ \\
\hline FOXH1 & $8 q 24.3$ & Low & NA & Heterozygous & (74) \\
\hline NODAL & $10 q 22.1$ & Low & NA & Heterozygous & $(75)$ \\
\hline GAS1 & $9 q 21.33$ & Low & Mainly inherited & Heterozygous & $(12)$ \\
\hline DLL1 & $6 q 27$ & Low & Inherited & Heterozygous & (15) \\
\hline CDON & $11 q 24.2$ & Low & NA & Heterozygous & (13) \\
\hline STIL & 1p33 & Low & Inherited & $\begin{array}{l}\text { Heterozygous } \\
\text { Homozygous }\end{array}$ & $(8,9,76)$ \\
\hline
\end{tabular}

Table 1. Characteristics of genes and mutations implicated in holoprosencephaly. 


\begin{tabular}{llc}
\hline Gene & Mutation & References \\
\hline \hline SHH & p.Gly290Asp & $(23)$ \\
ZIC2 & p.Ala461_Ala470dup & \\
SHH & p.Pro424Ala & $(23)$ \\
TGIF1 & del18p11 & \\
SHH & p.del378_380 & $(23)$ \\
TGIF1 & p.Thr151Ala & \\
GLI2 & p.Arg151Gly & $(70)$ \\
PTCH1 & p.Thr328Ala & \\
SIX3 & p.Ala93Asp & $(4)$ \\
PTCH1 & p.Ala393Thr & \\
SIX3 & p.Ala284Pro & $(77)$ \\
ZIC2 & p.Trp304Arg & \\
SHH & p.Leu218Pro & $(12)$ \\
GAS1 & p.Asp270Tyr & \\
SHH & p.Cys363Tyr & $(12)$ \\
GAS1 & p.Asp288Gly & \\
SHH & p.Pro347Gln & In this report \\
DISP1 & p.Met1096Thr & \\
\hline
\end{tabular}

\section{Table 2. Digenic inheritance in human HPE cases}


(a)

\begin{tabular}{|c|c|c|c|c|}
\hline & Sex & Age & Brain MRI & Face \\
\hline 11 & M & 81 & Microcephaly (-5SD) & - \\
\hline 12 & $\mathrm{~F}$ & 79 & - & - \\
\hline II1 & M & 48 & Microcephaly (-5SD) & - \\
\hline 112 & $\mathrm{~F}$ & 48 & - & - \\
\hline 113 & M & 46 & Microcephaly $(-4,5 \mathrm{SD})$ & Hypotelorism \\
\hline 115 & M & 42 & - & - \\
\hline 116 & $\mathrm{~F}$ & 35 & - & - \\
\hline III1 & $M$ (fetus) & - & Semi-lobar HPE & $\begin{array}{l}\text { Proboscis } \\
\text { Macroglossy }\end{array}$ \\
\hline III2 & F (fetus) & - & Alobar HPE & $\begin{array}{c}\text { Flat face } \\
\text { hypotelorism } \\
\text { Premaxillary agenesia } \\
\text { Cleft lip/palate }\end{array}$ \\
\hline III3 & F (fetus) & - & Severe HPE & NA \\
\hline III4 & $\mathrm{F}$ & 12 & - & - \\
\hline III5 & $\mathrm{F}$ & 11 & - & - \\
\hline 1116 & M & 12 & Microcephaly (-4SD) & $\begin{array}{l}\text { Hypotelorism } \\
\text { Cleft lip/palate }\end{array}$ \\
\hline III7 & M & 11 & Microcephaly (-3SD) & - \\
\hline 1118 & $\mathrm{~F}$ & 9 & - & - \\
\hline 1119 & $\mathrm{~F}$ & 6 & Microcephaly (-4SD) & - \\
\hline
\end{tabular}

(b)

\begin{tabular}{ccccc}
\hline & Sex & Age & Brain MRI & Face \\
\hline \hline $\mathbf{I 1}$ & M & 34 & - & - \\
\hline $\mathbf{1 2}$ & $\mathrm{F}$ & 33 & - & - \\
\hline & & & & Flat face \\
Short nose \\
Hypotelorism \\
Arched palate \\
Cleft palate \\
\end{tabular}

Tables 3. Phenotypic description of families F1 and F2 members. 


\begin{tabular}{|c|c|c|c|c|c|c|c|}
\hline \multirow[b]{2}{*}{ Gene } & \multirow[b]{2}{*}{$\begin{array}{l}\text { Nucleic } \\
\text { acid } \\
\text { change }\end{array}$} & \multirow[b]{2}{*}{$\begin{array}{l}\text { Amino acid } \\
\text { change }\end{array}$} & \multicolumn{4}{|c|}{ Minor Allele Frequencies } & \multirow[b]{2}{*}{$\begin{array}{c}\text { Bioinformatic } \\
\text { predictions }\end{array}$} \\
\hline & & & dbSNP & ESP6500 & $1000 G$ & ExAC & \\
\hline DISP1 & c. T3287C & p.Met1096Thr & rs144673025 & 0,005 & 0,001 & 0,006 & D:3 P:1 T:6 \\
\hline $\mathrm{SHH}$ & c.C1040A & p.Pro347Gln & - & 0 & 0 & 0 & D:9 P:1 T:0 \\
\hline DISP1 & c.A1087G & p.Asn363Asp & - & 0 & 0 & 0 & D:7 P:2 T:1 \\
\hline DISP1 & C.G1657A & p.Glu553Lys & - & 0 & 0 & 0 & D:5 P:1 T:4 \\
\hline
\end{tabular}

Table 4. Characteristics of DISP1 and SHH mutations found in F1 and F2 families 